This item was submitted to Loughborough's Research Repository by the author.

Items in Figshare are protected by copyright, with all rights reserved, unless otherwise indicated.

\title{
Separation coordinates, moduli spaces and Stasheff polytopes
}

PLEASE CITE THE PUBLISHED VERSION

http://dx.doi.org/10.1007/s00220-015-2332-x

PUBLISHER

(C) Springer Verlag

VERSION

AM (Accepted Manuscript)

\section{PUBLISHER STATEMENT}

This work is made available according to the conditions of the Creative Commons Attribution-NonCommercialNoDerivatives 4.0 International (CC BY-NC-ND 4.0) licence. Full details of this licence are available at: https://creativecommons.org/licenses/by-nc-nd/4.0/

\section{LICENCE}

CC BY-NC-ND 4.0

\section{REPOSITORY RECORD}

Schoebel, K., and Alexander Veselov. 2015. "Separation Coordinates, Moduli Spaces and Stasheff Polytopes". Loughborough University. https://hdl.handle.net/2134/16968. 


\title{
SEPARATION COORDINATES, MODULI SPACES AND STASHEFF POLYTOPES
}

\author{
K. SCHÖBEL AND A. P. VESELOV
}

\begin{abstract}
We show that the orthogonal separation coordinates on the sphere $S^{n}$ are naturally parametrised by the real version of the Deligne-MumfordKnudsen moduli space $\bar{M}_{0, n+2}(\mathbb{R})$ of stable curves of genus zero with $n+2$ marked points. We use the combinatorics of Stasheff polytopes tessellating $\bar{M}_{0, n+2}(\mathbb{R})$ to classify the different canonical forms of separation coordinates and deduce an explicit construction of separation coordinates as well as of Stäckel systems from the mosaic operad structure on $\bar{M}_{0, n+2}(\mathbb{R})$.
\end{abstract}

\section{INTRODUCTION}

Separation of variables is one of the oldest techniques in mathematical physics, which still remains one of the most effective and powerful tools in the theory of integrable systems. Its quantum version initiated by Lamé [17] is actually more natural than the classical one developed approximately at the same time by Jacobi [12], when one has to consider the Hamilton-Jacobi equation rather than the equations of motion.

The general theory of separation coordinates goes back to Stäckel [27] and LeviCivita [19] and was developed further by Eisenhart [10]. In the particular case of the sphere $S^{n}$, which we will be interested in, the first (and the most important) example, that of elliptic coordinates, was introduced already in 1859 by C. Neumann [23]. The classification problem of all separation coordinates on $S^{n}$ had been studied in detail by Olevski [24] and Kalnins \& Miller [13]. In particular, in the last paper to describe the answer the authors used a sophisticated graphical procedure similar to the one developed by Vilenkin to describe the polyspherical coordinates [30].

Despite all these advances almost nothing has been known about the global geometry of the space of separation coordinates. In the present paper we fill this gap by describing the topology and algebraic geometry of this space in the case of $S^{n}$. In particular, we link the graphical procedures from [13] and [30] with the rich combinatorial theory of associahedra, or Stasheff polytopes, introduced by Stasheff in 1963 in homotopy theory [28].

The reason this had not been done before is probably that the algebraic equations involved seemed far too complicated for a direct solution. Interestingly, Stäckel already commented on this in his habilitation thesis from 1891, where he notes [27, p. 6]:

Die Diskussion dieser Gleichungen ergab, dass es für $n=2$ drei wesentlich verschiedene Formen der Gleichung $H=0$ giebt, bei

1991 Mathematics Subject Classification. Primary 14H70, 14D21, 58D27; Secondary 14H10 .

Key words and phrases. Separation of variables, Stäckel systems, Stasheff polytopes, DeligneMumford-Knudsen moduli spaces. 
denen diese notwendigen Bedingungen erfüllt sind, und da diese Gleichungen auch wirklich Separation der Variabeln gestatten, ist die Frage für den Fall $n=2$ vollständig erledigt. Aber schon für $n=3$ werden die algebraischen Rechnungen so umständlich, dass mir eine weitere Verfolgung dieses Weges aussichtslos erschien. ${ }^{1}$

In a sense, our goal in this paper is to accomplish Stäckel's computations for arbitrary $n$, using a substantial progress made in the theory of moduli spaces in the last few decades. More precisely, we prove the following result.

Main Theorem. The Stäckel systems on $S^{n}$ with diagonal algebraic curvature tensor form a smooth projective variety isomorphic to the real Deligne-MumfordKnudsen moduli space $\bar{M}_{0, n+2}(\mathbb{R})$ of stable genus zero curves with $n+2$ marked points.

As a corollary we have that the set $X_{n}$ of equivalence classes of separation coordinates on the sphere $S^{n}$ modulo the orthogonal group is in one-to-one correspondence with the quotient space $Y_{n}=\bar{M}_{0, n+2}(\mathbb{R}) / S_{n+1}$. Since the real version of the Deligne-Mumford-Knudsen moduli space $\bar{M}_{0, n+2}(\mathbb{R})$ is known to be tessellated by $(n+1) ! / 2$ copies of the Stasheff polytope $K_{n}$ after Kapranov [15] and Devadoss [7], we can use the known results about $\bar{M}_{0, n+2}(\mathbb{R})$ and associahedra [8] to describe the combinatorial structure of $X_{n}$. In particular, we use the mosaic operad [7] to give an explicit construction for Stäckel systems and separation coordinates.

Note that in this way we establish (and exploit) a surprising correspondence between two seemingly completely unrelated objects - separation coordinates on a sphere on one hand and stable genus zero curves with marked points on the other hand - revealing yet another guise of the famous moduli space $\bar{M}_{0, n}(\mathbb{R})$.

The algebraic nature of the problem of separation of variables was explicitly revealed in [25], where the Nijenhuis integrability conditions for Killing tensors were reduced to purely algebraic equations for the associated algebraic curvature tensors. The statement of the Main Theorem stems from a thorough analysis of these equations in the first non-trivial case $n=3$, done in [26]. Its proof is based on the recent work by Aguirre, Felder and the second author [1], where the moduli space $\bar{M}_{0, n+2}$ was identified with the set of the Gaudin subalgebras in the KohnoDrinfeld Lie algebra $\mathfrak{t}_{n+1}$. We show that the Killing tensors on $S^{n}$ with diagonal algebraic curvature tensor satisfy the defining relations of the Kohno-Drinfeld Lie algebra, which allows us to make the link with the main result of [1].

\section{Separation coordinates, Killing tensors and StÄCkel systems}

2.1. Separation coordinates. Recall that the Hamilton-Jacobi equation

$$
\frac{1}{2} g^{i j} \frac{\partial W}{\partial x^{i}} \frac{\partial W}{\partial x^{j}}=E
$$

\footnotetext{
1 "For $n=2$ the discussion of these equations yielded three essentially different forms of the equation $H=0$ [the Hamilton-Jacobi equation], for which the necessary conditions are satisfied, and since these equations indeed allow a separation of variables, the question is completely settled in the case $n=2$. However, already for $n=3$ the algebraic computations become so cumbersome, that it seemed hopeless to me to pursue this approach further."
} 
separates in local coordinates $x^{1}, \ldots, x^{n}$ on a Riemannian manifold $M^{n}$ if it admits a solution of the form

$$
W\left(x^{1}, \ldots, x^{n} ; \underline{c}\right)=W_{1}\left(x^{1} ; \underline{c}\right)+\ldots+W_{n}\left(x^{n} ; \underline{c}\right), \quad \operatorname{det}\left(\frac{\partial^{2} W}{\partial x^{i} \partial c_{j}}\right) \neq 0,
$$

depending on $n$ parameters $\underline{c}=\left(c_{1}, \ldots, c_{n}\right)$. Note that if we reparametrise each coordinate $x^{i}$ with a strictly monotonic function $\Phi_{i}$, the Hamilton-Jacobi equation is still separable in the new coordinates $\Phi_{i}\left(x^{i}\right)$. The same is true for a permutation of the variables. In order to avoid this arbitrariness, we consider different coordinate systems as equivalent if they are related by such transformations. By abuse of language we will call a corresponding equivalence class simply separation coordinates. Equivalently, we can think of separation coordinates as the (unordered) system of coordinate hypersurfaces defined by the equations $x^{i}=$ constant. The separation coordinates are called orthogonal, if the normals of these hypersurfaces are mutually orthogonal.

The main tool in studying orthogonal separation coordinates are Killing tensors that satisfy a certain condition. The details of this relation will be explained in the rest of this section, with emphasis on spheres.

\subsection{Killing tensors.}

Definition 1. A Killing tensor on a Riemannian manifold $(M, g)$ is an element $K \in \Gamma\left(S^{2} T^{*} M\right)$ satisfying, in any coordinate system $x^{\alpha}(\alpha=1, \ldots, n)$, the equation

$$
\nabla_{\alpha} K_{\beta \gamma}+\nabla_{\beta} K_{\gamma \alpha}+\nabla_{\gamma} K_{\alpha \beta}=0
$$

where $\nabla$ is the Levi-Civita connection of the metric $g$.

Note that the metric $g$ is trivially a Killing tensor, because it is covariantly constant: $\nabla_{\alpha} g_{\beta \gamma}=0$. Here we will be concerned with Killing tensors on the standard round sphere $S^{n}$, regarded as the hypersurface

$$
S^{n}=\{x \in V:\|x\|=1\} \subset V
$$

of unit vectors in an $(n+1)$-dimensional Euclidean vector space $V$, equipped with the induced metric $g$.

Definition 2. An algebraic curvature tensor on a vector space $V$ is an element $R \in\left(V^{*}\right)^{\otimes 4}$ satisfying the usual (algebraic) symmetries of a Riemannian curvature tensor, namely:

$$
\begin{aligned}
& R_{j i k l}=-R_{i j k l}=R_{i j l k} \quad \text { (antisymmetry) } \\
& R_{k l i j}=R_{i j k l} \quad \text { (pair symmetry) } \\
& R_{i j k l}+R_{i k l j}+R_{i l j k}=0 \quad \text { (Bianchi identity). }
\end{aligned}
$$

The space of Killing tensors $K$ on $S^{n} \subset V$ is naturally isomorphic to the space of algebraic curvature tensors $R$ on $V$ [21]. This isomorphism is explicitly given by the formula

$$
K_{x}(v, w):=R(x, v, x, w)=\sum_{i, j, k, l=1}^{n+1} R_{i j k l} x^{i} x^{k} v^{j} w^{l}, \quad x \in S^{n}, \quad v, w \in T_{x} S^{n},
$$

where we consider a point $x \in S^{n}$ as well as the tangent vectors $v, w \in T_{x} S^{n}$ as vectors in $V$ satisfying $\|x\|=1$ and $v, w \perp x$. The above isomorphism is equivariant under the natural actions of the isometry group $\mathrm{O}(V)$ on Killing tensors and on algebraic curvature tensors respectively. 
2.3. Stäckel systems. In Definition 1, a Killing tensor is a symmetric bilinear form $K_{\alpha \beta}$ on the manifold $M$. In what follows we will interpret it in two other ways, each of which gives rise to a Lie bracket and hence to a Lie algebra generated by Killing tensors. On one hand, we can use the metric to identify the symmetric bilinear form $K_{\alpha \beta}$ with a symmetric endomorphism $K^{\alpha}{ }_{\beta}$. Interpreted in this way, the space of Killing tensors generates a Lie subalgebra of $\Gamma(\operatorname{End}(T M))$ with respect to the commutator bracket

$$
[K, L]=K L-L K
$$

On the other hand, we can interpret a Killing tensor $K_{\alpha \beta}$ as a function $K_{\alpha \beta} p^{\alpha} p^{\beta}$ on the total space of the cotangent bundle $T^{*} M$ which is quadratic in the fibres. Interpreted in this way, the space of Killing tensors generates a Lie subalgebra of $C^{\infty}\left(T^{*} M\right)$ with respect to the Poisson bracket

$$
\{K, L\}=\sum_{\alpha=1}^{n}\left(\frac{\partial K}{\partial x^{\alpha}} \frac{\partial L}{\partial p^{\alpha}}-\frac{\partial L}{\partial x^{\alpha}} \frac{\partial K}{\partial p^{\alpha}}\right) .
$$

Definition 3. A Stäckel system on an $n$-dimensional Riemannian manifold is an $n$-dimensional space of Killing tensors which mutually commute with respect to both of the following brackets:

(1) the commutator bracket (2.4)

(2) the Poisson bracket (2.5)

Remark 1. In the initial definition given by Stäckel, condition (2) is replaced by an integrability condition on the eigenspaces of the Killing tensors [27]. The equivalence of both definitions is proven in [2].

It can be shown that every Stäckel system contains a Killing tensor with simple eigenvalues [2]. Moreover, the $n$ distributions given by the orthogonal complements of its eigendirections are integrable. Hence they define $n$ hypersurface foliations with orthogonal normals or, equivalently, orthogonal coordinates. On the other hand, every Killing tensor commuting with the above and having simple eigenvalues defines the same coordinates. In this manner each Stäckel system defines a unique coordinate system. It is a classical result that these are separation coordinates and that every system of orthogonal separation coordinates arises in this way from a Stäckel system:

Theorem 1. [27, 10, 2] There is a bijective correspondence between Stäckel systems and orthogonal separation coordinates.

Remark 2. A priori, the above result is only a local result. However, any local Killing tensor field on a sphere can be extended to a global one (see e. g. [4]). Hence the same is true for the corresponding separation coordinates. That is why we can use the above result for a global classification of orthogonal separation coordinates on $S^{n}$.

2.4. Killing tensors with diagonal algebraic curvature tensor. In the following we will only consider Killing tensors on $S^{n}$ whose algebraic curvature tensor is diagonal in the following sense.

Definition 4. Due to the symmetries (2.2a) and (2.2b), we can interpret an algebraic curvature tensor $R$ on $V$ as a symmetric bilinear form on $\Lambda^{2} V$. We say that $R$ is diagonal in an orthonormal basis $\left\{e_{i}: 1 \leqslant i \leqslant n+1\right\}$ of $V$, if it is diagonal 
as a bilinear form on $\Lambda^{2} V$ in the associated basis $\left\{e_{i} \wedge e_{j}: 1 \leqslant i<j \leqslant n+1\right\}$. In components, this simply means that $R_{i j k l}=0$ unless $\{i, j\}=\{k, l\}$.

Restricting to Killing tensors with diagonal algebraic curvature tensor does not mean any loss of generality. The reason is the following refinement of Theorem 1 for spheres.

Theorem 2. [4] Necessary and sufficient conditions for the existence of an orthogonal separable coordinate system for the Hamilton-Jacobi equation on $S^{n}$ are that there are $n$ Killing tensors whose algebraic curvature tensors are diagonal in the same basis, one of which is the metric, which are linearly independent (locally) and pairwise commute with respect to the Poisson bracket.

Remark 3. By this theorem condition (2) in Definition 3 implies condition (1) on $S^{n}$. As we will show in Section 5 below, both conditions are actually equivalent for $S^{n}$.

The restriction to separation coordinates which are orthogonal does not constitute a loss of generality either, because of the following result.

Theorem 3. [13] All separation coordinates on $S^{n}$ are equivalent to orthogonal separation coordinates.

The equivalence corresponds to a linear change of the so-called ignorable coordinates, on which the metric does not depend (see [13]). We will consider separation coordinates up to this equivalence throughout this paper.

Theorems 2 and 3 reduce the classification of separation coordinates on spheres to the purely algebraic problem of finding certain abelian subalgebras in the following two Lie algebras.

Definition 5. We denote by $\mathfrak{d}_{n+1} \subset \Gamma\left(\operatorname{End}\left(T S^{n}\right)\right)$ and by $\mathscr{D}_{n+1} \subset C^{\infty}\left(T^{*} S^{n}\right)$ the Lie subalgebras generated by Killing tensors with diagonal algebraic curvature tensor under the commutator bracket (2.4) and the Poisson bracket (2.5) respectively.

By definition, a diagonal algebraic curvature tensor $R$ is uniquely determined by the diagonal elements $R_{i j i j}$ for $1 \leqslant i<j \leqslant n+1$. Indeed, the symmetries (2.2a) and (2.2b) determine the components $R_{i j i j}=-R_{i j j i}=R_{j i j i}=-R_{j i i j}$ for $i<j$. And if we set all other components to zero, the resulting tensor $R$ satisfies all symmetries (2.2) of an algebraic curvature tensor. For fixed $i$ and $j$, let $K_{i j}$ be the Killing tensor with diagonal algebraic curvature tensor $R$ given by

$$
R_{i j i j}=-R_{i j j i}=R_{j i j i}=-R_{j i i j}=1
$$

and all other components zero. Then $K_{i j}$ with $i<j$ form a basis of the space of Killing tensors on $S^{n}$ with diagonal algebraic curvature tensor and constitute a set of generators for both Lie algebras, $\mathfrak{d}_{n+1}$ and $\mathscr{D}_{n+1}$. The following two propositions show that they satisfy the same relations in $\mathfrak{d}_{n+1}$ and in $\mathscr{D}_{n+1}$.

Proposition 1. Let $K_{i j}, 1 \leqslant i<j \leqslant n+1$ be the basis of the space of Killing tensors on $S^{n}$ with diagonal algebraic curvature tensor, as defined above. For convenience we set $K_{j i}:=K_{i j}$. Then $K_{i j}$ satisfy the following relations in $\mathfrak{d}_{n+1}$ :

$$
\begin{aligned}
{\left[K_{i j}, K_{k l}\right] } & =0 & & \text { if } i, j, k, l \text { are distinct } \\
{\left[K_{i j}, K_{i k}+K_{j k}\right] } & =0 & & \text { if } i, j, k \text { are distinct. }
\end{aligned}
$$


Proof. We can extend the Killing tensor $K$ on $T_{x} S^{n}$ to a symmetric tensor $\hat{K}$ on $V=T_{x} S^{n} \oplus \mathbb{R} x$ by omitting the restriction $v, w \perp x$ in (2.3). The antisymmetry (2.2a) implies that $K_{x}(v, x)=0$ for any $v \in V$, so $\hat{K}$ is the extension of $K$ by zero. Consequently, we have $\left[\hat{K}_{i j}, \hat{K}_{k l}\right]=\left[{\widehat{K_{i j}, K_{k l}}}\right]$, so that it is sufficient to check the above relations on the corresponding extensions. To do so, consider the Killing tensor $K_{i j}$ at a point $x \in S^{n}$. By (2.3) and the definition (2.6) of the diagonal algebraic curvature tensor of $K_{i j}$ we have

$$
\begin{aligned}
\hat{K}_{i j}(v, w) & =\sum_{a, b=1}^{n+1}\left(R_{a b a b} x^{a} x^{a} v^{b} w^{b}+R_{a b b a} x^{a} x^{b} v^{b} w^{a}\right) \\
& =x^{i} x^{i} v^{j} w^{j}+x^{j} x^{j} v^{i} w^{i}-x^{i} x^{j} v^{i} w^{j}-x^{i} x^{j} v^{j} w^{i} .
\end{aligned}
$$

Let us put all indices down for convenience. Then we have

$$
\hat{K}_{i j}=\left(\begin{array}{cc}
x_{j}^{2} & -x_{i} x_{j} \\
-x_{i} x_{j} & x_{i}^{2}
\end{array}\right),
$$

where we left only non-zero ( $i$-th and $j$-th) rows and columns. This already proves relation $(2.7 \mathrm{a})$. To check the remaining relation $(2.7 \mathrm{~b})$, we compute

$$
\begin{aligned}
{\left[\hat{K}_{i j}, \hat{K}_{j k}\right] } & =\left[\left(\begin{array}{ccc}
x_{j}^{2} & -x_{i} x_{j} & 0 \\
-x_{i} x_{j} & x_{i}^{2} & 0 \\
0 & 0 & 0
\end{array}\right),\left(\begin{array}{ccc}
0 & 0 & 0 \\
0 & x_{k}^{2} & -x_{j} x_{k} \\
0 & -x_{j} x_{k} & x_{j}^{2}
\end{array}\right)\right] \\
& =x_{i} x_{j} x_{k}\left(\begin{array}{ccc}
0 & -x_{k} & x_{j} \\
x_{k} & 0 & -x_{i} \\
-x_{j} & x_{i} & 0
\end{array}\right) .
\end{aligned}
$$

Here we omitted rows and columns other than $i, j, k$, because they are zero. In the same way we compute $\left[\hat{K}_{i j}, \hat{K}_{i k}\right]$ and verify $(2.7 \mathrm{~b})$.

Proposition 2. As elements of $\mathscr{D}_{n+1}$ the generators $K_{i j}$ satisfy the following relations:

$$
\begin{aligned}
\left\{K_{i j}, K_{k l}\right\} & =0 & & \text { if } i, j, k, l \text { are distinct } \\
\left\{K_{i j}, K_{i k}+K_{j k}\right\} & =0 & & \text { if } i, j, k \text { are distinct. }
\end{aligned}
$$

Proof. The function on $T^{*} S^{n}$ given by $K_{i j}$ is

$$
K_{i j}(x, p)=x_{j}^{2} p_{i}^{2}+x_{i}^{2} p_{j}^{2}-2 x_{i} x_{j} p_{i} p_{j}=\left(x_{i} p_{j}-x_{j} p_{i}\right)^{2} .
$$

This already proves relation $(2.9 \mathrm{a})$. In order to verify relation $(2.9 \mathrm{~b})$, we compute

$$
\begin{aligned}
\left\{K_{i j}, K_{j k}\right\} & =\frac{\partial K_{i j}}{\partial x_{j}} \frac{\partial K_{j k}}{\partial p_{j}}-\frac{\partial K_{i j}}{\partial p_{j}} \frac{\partial K_{j k}}{\partial x_{j}} \\
& =4\left(x_{i} p_{j}-x_{j} p_{i}\right)\left(x_{j} p_{k}-x_{k} p_{j}\right)\left(x_{k} p_{i}-x_{i} p_{k}\right),
\end{aligned}
$$

which is clearly antisymmetric with respect to $i$ and $j$.

The next proposition says that there are no more relations between the generators and their brackets, both in $\mathfrak{d}_{n+1}$ as well as in $\mathscr{D}_{n+1}$, provided $n \geqslant 2$.

Proposition 3. The generators $K_{i j}$ and the commutator brackets $\left[K_{i j}, K_{j k}\right]$ with $1 \leqslant i<j<k \leqslant n+1$ are linearly independent in $\mathfrak{d}_{n+1}$ for $n \geqslant 2$. The same is true for $K_{i j}$ and the Poisson brackets $\left\{K_{i j}, K_{j k}\right\}$ as elements of $\mathscr{D}_{n+1}$. 
Proof. Since $K_{i j}$ are symmetric and $\left[K_{i j}, K_{j k}\right]$ are antisymmetric, it suffices to check the linear independence of both sets independently. The elements $K_{i j}$ are linearly independent by definition. To prove the linear independence of $\left[K_{i j}, K_{j k}\right]$ suppose that

$$
\sum_{1 \leqslant i<j<k \leqslant n+1} \lambda_{i j k}\left[K_{i j}, K_{j k}\right]=0 .
$$

For each triple $(p, q, r)$ with $1 \leqslant p<q<r \leqslant n+1$ consider a point $x \in S^{n}$ with $x_{m}=0$ if and only if $m \notin\{p, q, r\}$. Due to $(2.8)$ we have that $\left[K_{i j}, K_{j k}\right]=0$ at this point $x$ for all $i<j<k$ unless $(i, j, k)=(p, q, r)$. Hence all $\lambda_{p q r}=0$.

In the case of $\mathscr{D}_{n+1}$ we note that $K_{i j}$ are quadratic in momenta while $\left\{K_{i j}, K_{j k}\right\}$ are cubic; the rest of the proof is the same.

2.5. Stäckel systems generated from special conformal Killing tensors. The generic Stäckel systems on a sphere can be constructed from special conformal Killing tensors.

Definition 6. A special conformal Killing tensor on a Riemannian manifold is a symmetric tensor $L_{\alpha \beta}$ satisfying

$$
\nabla_{\gamma} L_{\alpha \beta}=\lambda_{\alpha} g_{\beta \gamma}+\lambda_{\beta} g_{\alpha \gamma} \quad \lambda=\frac{1}{2} \nabla \operatorname{tr} L .
$$

The space of special conformal Killing tensors parametrises geodesically equivalent metrics, which are metrics having the same set of unparametrised geodesics. Their importance in our context stems from the fact that

$$
K:=L-(\operatorname{tr} L) g
$$

defines a Killing tensor, as one immediately checks, and the fact that every Killing tensor of this form is contained in a Stäckel system. The latter follows easily from the Nijenhuis integrability conditions applied to $K$ (see for example [26]). Thus, in the generic case where $L$ (and hence $K$ ) has pairwise different eigenvalues, it defines a system of separation coordinates. The corresponding Stäckel system is spanned by the coefficients of the polynomial

$$
\operatorname{Adj}(L-\lambda \mathrm{Id})=\sum_{i=0}^{n-1} K_{i} \lambda^{i},
$$

where $\operatorname{Adj} X$ denotes the adjugate matrix, i. e. the transpose of the cofactor matrix of $X[3]$.

We can deduce the corresponding separation coordinates directly from the special conformal Killing tensor $L$, since its eigenvalues are constant on the corresponding coordinate hypersurfaces [5]. This means that the eigenvalues of $L$ can be taken as coordinate functions. On $S^{n} \subset V$ the situation is further simplified. The reason is that under certain conditions, which are met in this case, every special conformal Killing tensor $L$ on $M$ is the restriction of a covariantly constant symmetric tensor $\hat{L}$ on the metric cone over $M$ and vice versa (see for example [20]). Here the metric cone over $S^{n} \subset V$ is nothing but $V$, so the determination of separation coordinates on $S^{n} \subset V$ arising from a special conformal Killing tensor reduces to computing the eigenvalues of the restriction $L$ of a constant symmetric tensor $\hat{L}$ on $V$. 
2.6. Two extremal cases: elliptic and polyspherical coordinates. As a matter of example, let us consider two extremal cases. The generic case of orthogonal separation coordinates on the sphere consists of elliptic coordinates and can be obtained from a special conformal Killing tensor $L$ with simple eigenvalues as described above. For $\hat{L}$ with (constant) eigenvalues $\Lambda_{1}<\Lambda_{2}<\ldots<\Lambda_{n+1}$ the eigenvalues $\lambda_{1}(x), \ldots, \lambda_{n}(x)$ of $L$ at a point $x=\left(x_{1}, \ldots, x_{n+1}\right) \in S^{n}$ are the solutions of the equation

$$
\sum_{k=1}^{n+1} \frac{x_{k}^{2}}{\Lambda_{k}-\lambda}=0, \quad\|x\|^{2}=1
$$

which can be ordered to satisfy

$$
\Lambda_{1}<\lambda_{1}(x)<\Lambda_{2}<\lambda_{2}(x)<\cdots<\lambda_{n}(x)<\Lambda_{n+1} .
$$

This is nothing else but the defining equation for the classical elliptic coordinates on the sphere $S^{n}$ introduced in 1859 by C. Neumann [23]. Note that shifting or multiplying the parameters $\Lambda_{1}<\Lambda_{2}<\ldots<\Lambda_{n+1}$ by a constant results in a mere reparametrisation of the same coordinate system. Therefore elliptic coordinates form an $(n-1)$-parameter family of separation coordinates on $S^{n}$.

The other extreme, having no continuous parameters at all, are polyspherical coordinates considered by Vilenkin [30,31]. Each of these coordinate systems is given in terms of Cartesian coordinates by starting with $\boldsymbol{x}(\varnothing):=1$ on $S^{0} \subset \mathbb{R}^{1}$ and then defining recursively $\boldsymbol{z}=\boldsymbol{z}\left(\varphi_{1}, \ldots, \varphi_{n-1}\right)$ on $S^{n-1} \subset \mathbb{R}^{n}$ from $\boldsymbol{x}=\boldsymbol{x}\left(\varphi_{1}, \ldots, \varphi_{n_{1}-1}\right)$ on $S^{n_{1}-1} \subset \mathbb{R}^{n_{1}}$ and $\boldsymbol{y}=\boldsymbol{y}\left(\varphi_{n_{1}}, \ldots, \varphi_{n_{1}+n_{2}-2}\right)$ on $S^{n_{2}-1} \subset \mathbb{R}^{n_{2}}$ by setting

$$
\boldsymbol{z}=\left(\boldsymbol{x} \cos \varphi_{n-1}, \boldsymbol{y} \sin \varphi_{n-1}\right)
$$

for $n=n_{1}+n_{2}$. Since this involves a choice of a splitting $n=n_{1}+n_{2}$ in each step, polyspherical coordinates on $S^{n-1}$ are parametrised by planar rooted binary trees with $n$ leaves. ${ }^{2}$ For example, the standard spherical coordinates correspond to the binary tree where each right child is a leaf.

2.7. The residual action of the isometry group. Theorem 2 implies that for a Stäckel system on a sphere we can always find an isometry which takes all Killing tensors in this Stäckel system to Killing tensors having a diagonal algebraic curvature tensor. This means that the space of Killing tensors with diagonal algebraic curvature tensor defines a slice for the action of the isometry group. If we want to classify separation coordinates up to isometries, we have to take into account that the stabiliser of this slice in the isometry group is not trivial.

Due to the symmetries $(2.2 \mathrm{a})$ and $(2.2 \mathrm{~b})$, the space of algebraic curvature tensors is a subspace of the space $S^{2} \Lambda^{2} V$ of symmetric forms on $\Lambda^{2} V$. The natural action of the isometry group $\mathrm{O}(V)$ on this space is given as follows. Mapping an orthonormal basis $\left\{e_{i}: 1 \leqslant i \leqslant n+1\right\}$ of $V$ to the basis $\left\{\left(e_{i} \wedge e_{j}\right) / \sqrt{2}: 1 \leqslant i<j \leqslant n+1\right\}$ of $\Lambda^{2} V$ defines a map

$$
\mathrm{O}(V) \rightarrow \mathrm{O}\left(\Lambda^{2} V\right)
$$

\footnotetext{
${ }^{2}$ When Vilenkin introduced polyspherical coordinates in [30], he used trees which are not binary. The description with binary trees appeared in Vilenkin and Klimyk [31, Chap. 10.5] and both are completely equivalent.
} 
since the latter basis is orthonormal with respect to the scalar product on $\Lambda^{2} V$ induced from the one on $V$. Via the action of $\mathrm{O}\left(\Lambda^{2} V\right)$ on $\Lambda^{2} V$ this defines an action of $\mathrm{O}(V)$ on $S^{2} \Lambda^{2} V$ and hence on algebraic curvature tensors.

In general, the subgroup in $\mathrm{O}(V)$ leaving the space of diagonal bilinear forms on $V$ invariant is the subgroup of signed permutation matrices, acting by permutations and sign changes of the chosen basis $\left\{e_{i}\right\}$ in $V$. This group is the symmetry group of the hyperoctahedron in $V$ with vertices $\pm e_{i}$ and is isomorphic to the semidirect product $S_{N} \ltimes \mathbb{Z}_{2}^{N}$, where $N=\operatorname{dim} V=n+1$.

The stabiliser in $\mathrm{O}(V)$ of the space of diagonal algebraic curvature tensors is now the preimage under (2.14) of the stabiliser of diagonal bilinear forms on $\Lambda^{2} V$. Since the latter consists of permutations and sign changes of the basis $\left\{e_{i} \wedge e_{j}\right\}$ in $\Lambda^{2} V$, this is just the group of permutations and sign changes of the basis elements $e_{i}$, i. e. the group described in the preceding paragraph. Note that the normal subgroup of sign changes, which is isomorphic to $\mathbb{Z}_{2}^{N}$, acts trivially on diagonal bilinear forms. Hence the action descends to the quotient $\left(S_{N} \ltimes \mathbb{Z}_{2}^{N}\right) / \mathbb{Z}_{2}^{N} \cong S_{N}$. Summarising the above, we have:

Proposition 4. The stabiliser in the isometry group $\mathrm{O}(V)$ of the space of Killing tensors on $S^{n} \subset V$ with diagonal algebraic curvature tensor is the hyperoctahedral group and isomorphic to the semidirect product $S_{N} \ltimes \mathbb{Z}_{2}^{N}$, where $N=n+1$. This action descends to an action of $S_{N}$ given by

$$
\sigma\left(K_{i j}\right)=K_{\sigma(i) \sigma(j)}, \quad \sigma \in S_{N} .
$$

\section{Gaudin subalgebras of the Kohno-Drinfeld Lie algebra and the MODULI SPACE $\bar{M}_{0, n+1}$}

We describe now the result of [1], which plays a key role for us. The (real version of) the Kohno-Drinfeld Lie algebra $\mathfrak{t}_{n}(n=2,3, \ldots)$ is defined as the quotient of the free Lie algebra over $\mathbb{R}$ with generators $t_{i j}=t_{j i}, i \neq j \in\{1, \ldots, n\}$, by the ideal generated by the relations

$$
\begin{aligned}
{\left[t_{i j}, t_{k l}\right] } & =0, & & \text { if } i, j, k, l \text { are distinct, } \\
{\left[t_{i j}, t_{i k}+t_{j k}\right] } & =0, & & \text { if } i, j, k \text { are distinct. }
\end{aligned}
$$

This Lie algebra appeared in Kohno's work as the holonomy Lie algebra of the complement to the union of the diagonals $z_{i}=z_{j}, i<j$ in $\mathbb{C}^{n}$ (which is also a configuration space of $n$ distinct points on the complex plane) and in Drinfeld's work as the value space of the universal Knizhnik-Zamolodchikov connection (see the references in [1]).

Gaudin subalgebras of Kohno-Drinfeld Lie algebras were introduced in [1] as the abelian Lie subalgebras of maximal dimension contained in the linear span $\mathfrak{t}_{n}^{1}$ of the generators $t_{i j}$. The main class of examples is provided by Gaudin's models of integrable spin chains

$$
\mathfrak{g}_{n}(z)=\left\{\sum_{1 \leqslant i<j \leqslant n} \frac{b_{i}-b_{j}}{z_{i}-z_{j}} t_{i j}, \quad b \in \mathbb{R}^{n}\right\} .
$$

Note that they are parametrised by $z \in \Sigma_{n} /$ Aff, where

$$
\Sigma_{n}=\mathbb{R}^{n} \backslash \bigcup_{i<j}\left\{z \in \mathbb{R}^{n} \mid z_{i}=z_{j}\right\}
$$


is the configuration space of $n$ distinct ordered points on the real line and Aff is the group of affine maps $z \mapsto a z+b, a \neq 0$, acting diagonally on $\mathbb{R}^{n}$. A different type of example, which came from the representation theory of the symmetric group, is given by the Jucys-Murphy subalgebras spanned by

$$
t_{12}, \quad t_{13}+t_{23}, \quad t_{14}+t_{24}+t_{34}, \quad \ldots
$$

(see [29] and references therein).

The main result of [1] is the following.

Theorem 4. [1] Gaudin subalgebras in $\mathfrak{t}_{n}$ form a nonsingular algebraic subvariety of the Grassmannian $G(n-1, n(n-1) / 2)$ of $(n-1)$-dimensional subspaces in $\mathfrak{t}_{n}^{1}$, isomorphic to the moduli space $\bar{M}_{0, n+1}$ of stable curves of genus zero with $n+1$ marked points.

In fact, the result holds for any quotient of $\mathfrak{t}_{n}$ where both the generators $t_{i j}$, $1 \leqslant i<j \leqslant n$, and the brackets $\left[t_{i j}, t_{j k}\right], 1 \leqslant i<j<k \leqslant n$, are linearly independent (see remark 2.6 in [1]), and over any field.

The most popular version of the moduli space $\bar{M}_{0, n+1}$ - appearing, for example, in the celebrated Witten's conjecture - is defined over $\mathbb{C}$. It is a particular (DeligneMumford) compactification of the configuration space $M_{0, n+1}(\mathbb{C})$ of $n+1$ distinct labelled points in $\mathbb{C} P^{1}$ modulo $P G L_{2}(\mathbb{C})$ studied by Knudsen [16], who proved that it is a smooth projective variety. The compactification $\bar{M}_{0, n+1}(\mathbb{C})$ includes the singular rational curves with double point singularities and with the following properties: the graph of components is a tree (genus zero) and each irreducible component contains at least three marked or singular points (stability condition).

However, we need the real version $\bar{M}_{0, n+1}(\mathbb{R})$, which we discuss next.

\section{The Real version $\bar{M}_{0, n+1}(\mathbb{R})$ and Stasheff polytopes}

4.1. Topology. The real version $\bar{M}_{0, n+1}(\mathbb{R})$ was studied in more detail by Kapranov [15] and Devadoss [7]. By Knudsen's theorem, which works over $\mathbb{R}$ as well, $\bar{M}_{0, n+1}(\mathbb{R})$ is a smooth real manifold of dimension $n-2$. It can be described as an iterated blow-up of $\mathbb{R} P^{n-2}[15,7,6] . \bar{M}_{0,4}(\mathbb{R})$ is simply $\mathbb{R} P^{1}$ and $\bar{M}_{0,5}(\mathbb{R})$ is a non-orientable surface with Euler characteristic -3 , which is a connected sum of five copies of $\mathbb{R} P^{2}$.

The topology of $\bar{M}_{0, n+1}(\mathbb{R})$ becomes increasingly complicated when $n$ grows. It is known to be aspherical (Davis et al. [6]). The Euler characteristic can be given explicitly by

$$
\chi\left(\bar{M}_{0, n+1}(\mathbb{R})\right)=(-1)^{\frac{n-2}{2}}(n-1) ! !(n-3) ! !
$$

for even $n$ (and zero for odd $n$ ), see [7]. A description of the cohomology is more complicated than in the complex case, as found by Etingof et al. in [11].

4.2. Combinatorics. Fortunately, a lot of information about $\bar{M}_{0, n+1}(\mathbb{R})$ is encapsulated in a well studied remarkable polytope known as associahedron, or Stasheff polytope $K_{n}$. Namely, $\bar{M}_{0, n+1}(\mathbb{R})$ is tessellated by $n ! / 2$ copies of $K_{n}$, see $[15,7]$.

$K_{n}$ was first described by Stasheff as a combinatorial object in the homotopy theory of $H$-spaces [28] (see the history of this in Stasheff's contribution to [22]). Its first realisation as a convex polytope is usually ascribed to Milnor. By now we have several geometric realisations of Stasheff polytopes, see e.g. [9] and references therein. $\quad K_{n}$ is a convex polytope of dimension $n-2: K_{3}$ is a segment, $K_{4}$ is 
a pentagon and $K_{5}$ is the polyhedron shown in Figure 1, which can be obtained combinatorially by cutting off three skew edges from a cube.

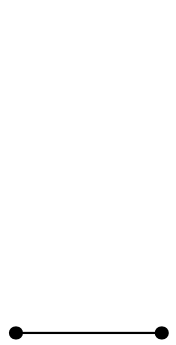

(a) $K_{3}$

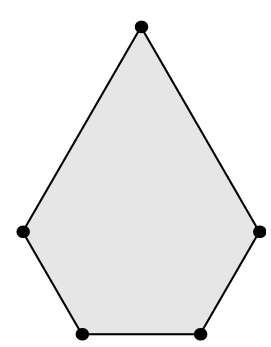

(b) $K_{4}$

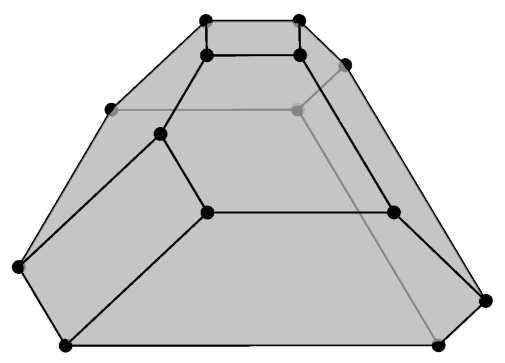

(c) $K_{5}$

FigURE 1. Stasheff polytopes.

The faces of $K_{n}$ of codimension $d$ are in one-to-one correspondence with dissections of a based $(n+1)$-gon by $d$ non-intersecting diagonals (see e.g. [8]). In particular, the vertices of $K_{n}$ correspond to the triangulations of the $(n+1)$-gon by non-intersecting diagonals and their number is $C_{n-1}$, where

$$
C_{n}=\frac{1}{n+1}\left(\begin{array}{c}
2 n \\
n
\end{array}\right)
$$

is the Catalan number.

Alternatively, the faces of $K_{n}$ can be labelled by non-isomorphic planar rooted trees with $n$ leaves (see e.g. [7]). These are simply the dual graphs of the dissected polygons, cut off at the edges of the polygon. In particular, the vertices of $K_{n}$ correspond to binary rooted trees. For $n=4$ this is depicted in Figure 2.
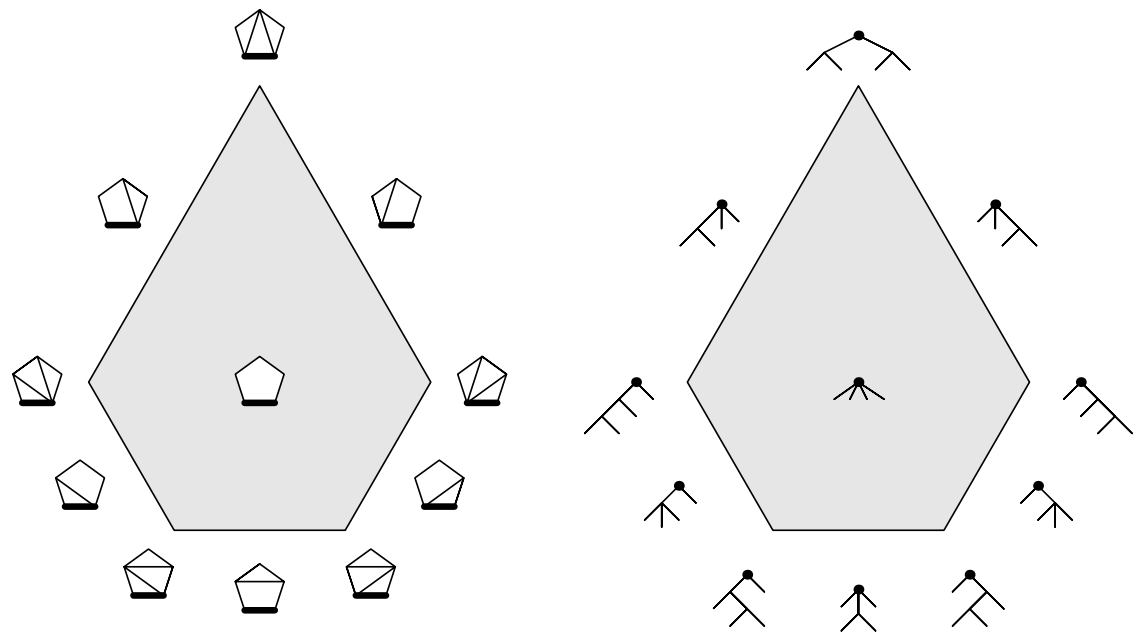

Figure 2. Labellings of $K_{4}$ by dissections of a based pentagon (left) and planar rooted trees with four leaves (right).

The Stasheff polytope $K_{n}$ admits a realisation with the dihedral symmetry $D_{n+1}$, which is the symmetry group of a regular $(n+1)$-gon [18]. 
4.3. Operad structure. The sequence of moduli spaces $\bar{M}_{0, n+1}(\mathbb{R})$ for $n=1,2, \ldots$. carries a natural operad structure, called the "mosaic operad" [7].

Definition 7. An operad structure on a sequence of objects $\mathcal{O}(n)$ is a composition map

$$
\begin{aligned}
& \circ: \quad \mathcal{O}(k) \times \mathcal{O}\left(n_{1}\right) \times \cdots \times \mathcal{O}\left(n_{k}\right) \longrightarrow \mathcal{O}\left(n_{1}+\cdots+n_{k}\right) \\
& \left(y, x_{1}, \ldots, x_{k}\right) \mapsto y \circ\left(x_{1}, \ldots, x_{k}\right)
\end{aligned}
$$

together with a right action

$$
\begin{aligned}
\star \mathcal{O}(n) \times S_{n} & \longrightarrow \mathcal{O}(n) \\
(x, \pi) & \mapsto x \star \pi
\end{aligned}
$$

of the permutation group $S_{n}$ on each object $\mathcal{O}(n)$, satisfying the following axioms:

Identity: There is a distinguished element $1 \in \mathcal{O}(1)$ satisfying

$$
y \circ(1, \ldots, 1)=y=1 \circ y \text {. }
$$

Associativity: For $z \in \mathcal{O}(k), y_{i} \in \mathcal{O}\left(n_{i}\right), i=1, \ldots, k$, and $x_{i} \in \mathcal{O}\left(m_{i, 1}\right) \times$ $\cdots \times \mathcal{O}\left(m_{i, n_{i}}\right)$ we have

$$
z \circ\left(y_{1} \circ x_{1}, \ldots, y_{k} \circ x_{k}\right)=\left(z \circ\left(y_{1}, \ldots, y_{k}\right)\right) \circ\left(x_{1}, \ldots, x_{k}\right) .
$$

\section{Equivariance:}

$$
\begin{aligned}
(y \star \pi) \circ\left(x_{1}, \ldots, x_{k}\right) & =y \circ\left(\left(x_{1}, \ldots, x_{k}\right) \star \pi\right) \\
y \circ\left(x_{1} \star \pi_{1}, \ldots, x_{k} \star \pi_{k}\right) & =\left(y \circ\left(x_{1}, \ldots, x_{k}\right)\right) \star\left(\pi_{1}, \ldots, \pi_{k}\right),
\end{aligned}
$$

where $S_{k}$ acts on $\left(x_{1}, \ldots, x_{k}\right)$ by permutation and $\left(\pi_{1}, \ldots, \pi_{k}\right)$ on $\mathcal{O}\left(n_{1}+\right.$ $\left.\cdots+n_{k}\right)$ under the inclusion $S_{n_{1}} \times \cdots \times S_{n_{k}} \hookrightarrow S_{n_{1}+\cdots+n_{k}}$.

In terms of dissected polygons, the operad structure on $\mathcal{O}(n):=\bar{M}_{0, n+1}(\mathbb{R})$ is given by gluing the $k\left(n_{i}+1\right)$-gons $x_{i}$ with their base to the $k$ non-base edges of the $(k+1)$-gon $y$ to form the $\left(n_{1}+\ldots+n_{k}+1\right)$-gon $y \circ\left(x_{1}, \ldots, x_{k}\right)$ with the base of $y$ as base. If $y$ is dissected by $d_{0}$ diagonals and $x_{i}$ by $d_{i}$ diagonals, then $y \circ\left(x_{1}, \ldots, x_{k}\right)$ is dissected by $d_{0}+d_{1}+\cdots d_{k}+k$ diagonals, namely the diagonals of $y$ and $x_{1}, \ldots, x_{k}$ plus the $k$ glued pairs of edges which become diagonals after gluing. On planar trees, the composition $y \circ\left(x_{1}, \ldots, x_{k}\right)$ is given by grafting the $k$ trees $x_{i}$ with their root to the leaves of the tree $y$.

The operad structure on Stasheff polytopes defines a map

$$
\circ: K_{k} \times K_{n_{1}} \times \cdots \times K_{n_{k}} \hookrightarrow K_{n_{1}+\cdots+n_{k}}
$$

whose image is a codimension $k$ face of $K_{n_{1}+\cdots+n_{k}}$. This yields a decomposition of the faces of a Stasheff polytope into products of Stasheff polytopes [28].

\section{THE CORRESPONDENCE}

After these preparations we are now in a position to state our main result. By Propositions 1 and 2 the defining relations of the Kohno-Drinfeld Lie algebra $t_{n}$ are satisfied in the Lie algebras $\mathfrak{d}_{n}$ and $\mathscr{D}_{n}$ (c.f. Definition 5 ). This provides surjective Lie algebra morphisms

$$
\mathfrak{t}_{n} \longrightarrow \mathfrak{d}_{n} \quad \mathfrak{t}_{n} \longrightarrow \mathscr{D}_{n},
$$

given by mapping the generator $t_{i j}$ to the Killing tensor $K_{i j}$. Under these morphisms the linear span $\mathfrak{t}_{n}^{1}$ of the $t_{i j}$ is isomorphic to the space of Killing tensors - interpreted as endomorphisms in $\mathfrak{d}_{n}$ respectively as quadratic functions on the 
cotangent bundle in $\mathscr{D}_{n}$. Thus the above morphisms map Gaudin subalgebras in the Kohno-Drinfeld Lie algebra $\mathfrak{t}_{n}$ to Stäckel systems on $S^{n-1}$ with diagonal algebraic curvature tensor. Proposition 3 now shows that this defines an isomorphism between Gaudin subalgebras and Stäckel systems. Now using Theorem 4 we have the following correspondence:

Theorem 5. The Stäckel systems on $S^{n}$ with diagonal algebraic curvature tensor form a nonsingular algebraic subvariety of the Grassmannian $G(n, n(n+1) / 2)$ of $n$-planes in the space of Killing tensors with diagonal algebraic curvature tensor, which is isomorphic to the real Deligne-Mumford-Knudsen moduli space $\bar{M}_{0, n+2}(\mathbb{R})$ of stable genus zero curves with $n+2$ marked points.

Note that since $\bar{M}_{0, n+2}(\mathbb{R})$ can be considered as a compactification of the configuration space $\Sigma_{n+1} /$ Aff of $n+1$ ordered distinct points on a real line modulo the affine group, we have a natural action of the symmetric group $S_{n+1}$ on $\bar{M}_{0, n+2}(\mathbb{R})$.

Corollary 1. The space $X_{n}$ of equivalence classes of orthogonal separation coordinates on the sphere $S^{n}$ modulo the orthogonal group $O(n+1)$ is naturally homeomorphic to the quotient space $Y_{n}=\bar{M}_{0, n+2}(\mathbb{R}) / S_{n+1}$.

Proof. This follows directly from Theorems 1 and 5 . It suffices to note that by Proposition 4 the morphisms (5.1) are equivariant with respect to the $S_{n+1}$-action on $\mathfrak{t}_{n+1}$ and $\mathfrak{d}_{n+1}$ respectively $\mathscr{D}_{n+1}$. Therefore the isomorphism in Theorem 5 is $S_{n+1}$-equiviariant, so that the corresponding quotients are homeomorphic. Note that while the space of Stäckel systems on $S^{n} \subset V$ with diagonal algebraic curvature tensor depends on the choice of an orthonormal basis in the ambient space $V$ (for which the algebraic curvature tensors are diagonal), the quotient does not. Therefore the homeomorphism is natural.

Since $\bar{M}_{0, n+2}(\mathbb{R})$ is tessellated by $(n+1) ! / 2$ copies of the Stasheff polytope $K_{n+1}$, we can use it to describe the quotient. The interior of $K_{n+1}$ corresponds to the classical elliptic coordinates (2.12) on the sphere $S^{n}$. The $n+1$ distinct real parameters $\left(\Lambda_{1}, \ldots, \Lambda_{n+1}\right) \in \Sigma_{n+1}$ they depend on are the eigenvalues of the symmetric tensor $\hat{L}$ on $\mathbb{R}^{n+1}$ which restricts to the corresponding special conformal Killing tensor $L$ on $S^{n}$. Shifting or scaling them only reparametrises the corresponding coordinates. Hence the actual parameter space is the quotient $\Sigma_{n+1} /$ Aff, which is nothing else but the open moduli space $M_{0, n+2}(\mathbb{R})$. Thus we have the following important

Corollary 2. All orthogonal separation coordinates on $S^{n}$ belong to the closure of the Neumann family of elliptic coordinates. The possible degenerations of the Neumann family correspond to the faces of the Stasheff polytope $K_{n+1}$.

The first part is probably not surprising for the experts (see the similar claim in the complex case in [14]), but we have not seen it explicitly stated and proved in the literature. In Section 6.2 we will show that rather than by actually performing the limiting process explicitly (as in [14]), the limiting cases can be better understood by composing generic separation coordinates (that is elliptic coordinates) of lower dimensions under the operad composition. The same holds true for the corresponding Stäckel systems.

Because we have $(n+1) ! / 2$ Stasheff polytopes $K_{n+1}$ tiling $\bar{M}_{0, n+2}(\mathbb{R})$, the quotient $Y_{n}=\bar{M}_{0, n+2}(\mathbb{R}) / S_{n+1}$ is actually only "a half" of $K_{n+1}$ with some identification between the faces. In the interior of the polytope the identification is 
given by the action of $\mathbb{Z}_{2} \subset D_{n+2}$, corresponding to a reflection in the dihedral group, realised as an isometry of $K_{n+1}$ (see above). If we are using the blowup description of $\bar{M}_{0, n+2}(\mathbb{R})[15,7,6]$, then it corresponds to the longest element $(1,2, \ldots, n, n+1) \mapsto(n+1, n, \ldots, 2,1)$ in the symmetric group $S_{n+1}$, mapping the $A_{n}$ Weyl chamber into the opposite one. For $K_{4}$ this is just a reflection symmetry of the pentagon as indicated in Figure 4.

The identification of the faces is more sophisticated. Probably the best way to describe them is using the "twisting along the diagonal" operation for the dissected $(n+1)$-gon introduced in $[7,8]$. On planar trees this corresponds to reversing the ordering of a node's children. Trees which are equivalent under this operation are called "dyslexic".

As a matter of illustration, let us consider the least non-trivial example $n=$ 2, depicted in Figure 3 . The parameter space $\bar{M}_{0,4}(\mathbb{R}) \cong \mathbb{R} P^{1}$ is just a circle,

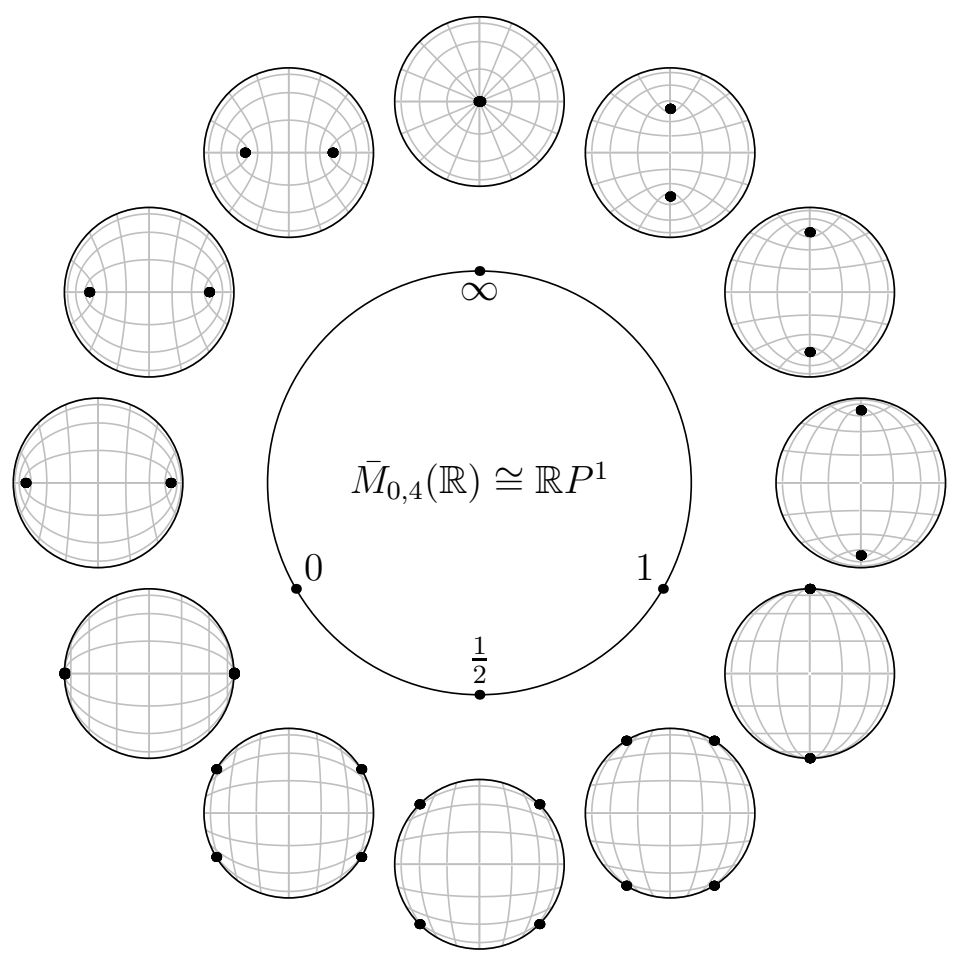

Figure 3. Orthogonal separation coordinates on $S^{2}$ parametrised by $\bar{M}_{0,4}(\mathbb{R}) \cong \mathbb{R} P^{1}$.

parametrised by the affine-invariant cross ratio $\tau=\frac{\Lambda_{2}-\Lambda_{1}}{\Lambda_{3}-\Lambda_{1}}$. It is tessellated by three copies of the Stasheff polytope $K_{3}$, the tiles being the arcs between the points $\tau=0,1, \infty$. We can identify $K_{3}$ with the arc [0,1], for which $\Lambda_{1}<\Lambda_{2}<\Lambda_{3}$. The symmetry group $S_{3}$ acts by permuting $\Lambda_{1}, \Lambda_{2}$ and $\Lambda_{3}$ and hence the points 0,1 and $\infty$. The quotient $Y_{2}=\bar{M}_{0,4}(\mathbb{R}) / S_{3}$ can be identified with the arc $\left[0, \frac{1}{2}\right]$. This is "a half" of $K_{3}$, where $\tau=0$ corresponds to spherical coordinates and $\tau=1 / 2$ to the "lemniscatic" case, which is just a particular case of elliptic coordinates when 
$\Lambda_{2}=\left(\Lambda_{1}+\Lambda_{3}\right) / 2$. In this example there is no identification in place, because the dimension is too low.

\section{Applications}

6.1. Enumerating separation coordinates. For a Stasheff polytope, the number of non-equivalent faces of a given dimension can be given by the following Devadoss-Read formula [8]. Let $A(x, y)=\sum a_{m n} x^{m} y^{n}$ be the formal series solution of the functional equation

$$
A(x, y)=y+\frac{1}{2}\left(\frac{A(x, y)^{2}}{1-A(x, y)}+\frac{(1+A(x, y)) A\left(x^{2}, y^{2}\right)}{1-A\left(x^{2}, y^{2}\right)}\right) .
$$

There is no closed formula for $A(x, y)$, but one uses Equation (6.1) to find the coefficients $a_{m n}$ recursively. The claim is that the coefficient $a_{m n}$ is the number of non-equivalent faces of $K_{n}$ of codimension $m-1$. Devadoss and Read proved this using a combinatorial technique going back to Pólya [8].

Using Table 2 from [8], we get the number of non-equivalent canonical forms of separation coordinates on $S^{n}$ for $n \leqslant 10$, as listed in Table 1. The 1's on the

TABle 1. Number of canonical forms for separation coordinates on $S^{n}$, ordered by increasing number of independent continuous parameters.

\begin{tabular}{r|rrrrrrrrrr|r}
\hline & 0 & 1 & 2 & 3 & 4 & 5 & 6 & 7 & 8 & 9 & total \\
\hline$S^{2}$ & 1 & 1 & & & & & & & & 2 \\
$S^{3}$ & 2 & 3 & 1 & & & & & & & 6 \\
$S^{4}$ & 3 & 8 & 5 & 1 & & & & & & 17 \\
$S^{5}$ & 6 & 20 & 22 & 8 & 1 & & & & & 57 \\
$S^{6}$ & 11 & 49 & 73 & 46 & 11 & 1 & & & & 191 \\
$S^{7}$ & 23 & 119 & 233 & 206 & 87 & 15 & 1 & & & 684 \\
$S^{8}$ & 46 & 288 & 689 & 807 & 485 & 147 & 19 & 1 & & 2482 \\
$S^{9}$ & 98 & 696 & 1988 & 2891 & 2320 & 1021 & 236 & 24 & 1 & 9275 \\
$S^{10}$ & 207 & 1681 & 5561 & 9737 & 9800 & 5795 & 1960 & 356 & 29 & 1 & 35127 \\
\hline
\end{tabular}

diagonal correspond to elliptic coordinates, the numbers in the first column to polyspherical coordinates. Note that the sequence $1,2,3,6,11,23, \ldots$ is the sequence of Wedderburn-Etherington numbers, i. e. the number of non-planar binary rooted trees with $n+1$ leaves [32]. This reflects the fact that we can parametrise polyspherical coordinates by planar binary rooted trees and that the notions dyslexic and non-planar coincide for binary trees.

In the first row we have 1 and 1, corresponding to spherical and elliptic coordinates on $S^{2}$ respectively, as discussed in Section 5. The numbers in the second row - 2, 3, 1 - are in perfect agreement with the results of Eisenhart [10], Olevski [24] and Kalnins \& Miller [13]. They correspond to spherical and cylindrical coordinates (2), two types of Lamé rotational coordinates plus Lamé subgroup reduction (3) and elliptic coordinates (1) respectively. Their identification with the faces of the Stasheff polytope $K_{4}$ is indicated in Figure 4, in comparison to the different labellings shown in Figure 2. Polyspherical coordinates comprise, for example, the usual spherical coordinates plus cylindrical coordinates. Note that "the half" of $K_{4}$, 


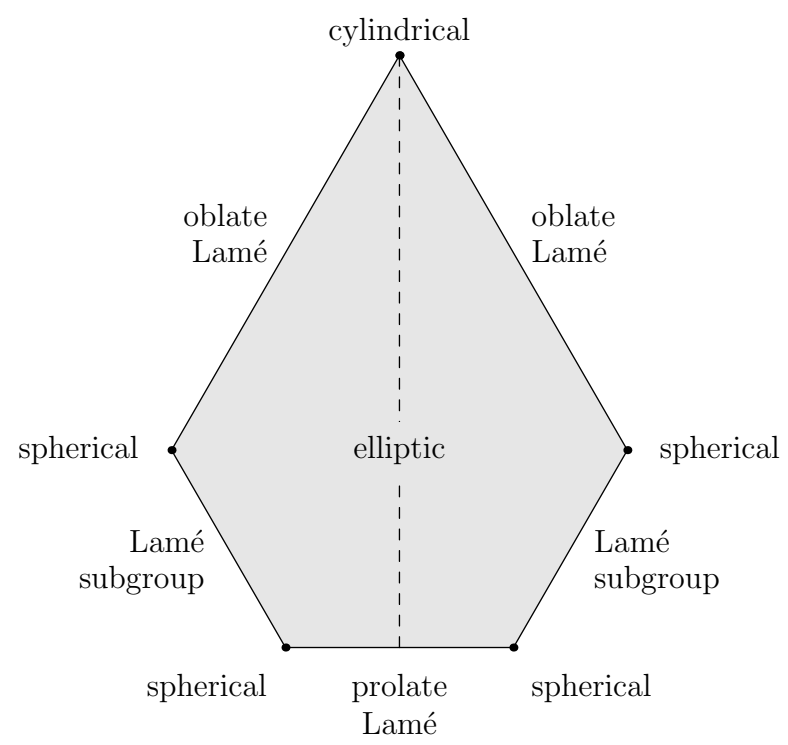

Figure 4 . The Stasheff polytope $K_{4}$, labelled by separation coordinates on $S^{3}$.

obtained as the quotient under the reflection symmetry indicated in Figure 4, is a quadrilateral and that for the quotient space $Y_{3}=\bar{M}_{0,5}(\mathbb{R}) / S_{4}$ we have to identify its two adjacent vertices that are labelled by spherical coordinates and joined by Lamé subgroup reduction.

In accordance with our description, the total number of canonical forms for separation coordinates on $S^{n}$, indicated in the last column in Table 1, is the number of dyslexic planar rooted trees with $n+1$ leaves [33].

6.2. Constructing separation coordinates via the mosaic operad. The correspondence in Theorem 5 transfers the natural operad structure on $\bar{M}_{0, n+2}(\mathbb{R})$ to orthogonal separation coordinates on $S^{n}$ and thereby yields a uniform construction procedure for separation coordinates on spheres. Implicitly this structure is already present in [13] (see, in particular, formula (3.14) therein). Let us now make this operad structure explicit. We begin with the operad structure on $\mathcal{O}(n):=\mathbb{R}^{n}$ given by

$$
\begin{aligned}
& \circ: \mathbb{R}^{k} \times \mathbb{R}^{n_{1}} \times \ldots \times \mathbb{R}^{n_{k}} \longrightarrow \mathbb{R}^{n_{1}+\cdots+n_{k}} \\
& \left(\boldsymbol{y}, \boldsymbol{x}_{1}, \ldots, \boldsymbol{x}_{k}\right) \quad \mapsto \boldsymbol{y} \circ\left(\boldsymbol{x}_{1}, \ldots, \boldsymbol{x}_{k}\right):=\left(y_{1} \boldsymbol{x}_{1}, \ldots, y_{k} \boldsymbol{x}_{k}\right)
\end{aligned}
$$

together with the permutation action of $S_{n}$ on $\mathbb{R}^{n}$. This operad structure descends to an operad structure on $\mathcal{O}(n):=S^{n-1} \subset \mathbb{R}^{n}$, since $\left(y_{1} \boldsymbol{x}_{1}\right)^{2}+\cdots\left(y_{k} \boldsymbol{x}_{k}\right)^{2}=$ $y_{1}^{2}+\cdots y_{k}^{2}=1$ for $\boldsymbol{y} \in S^{k-1}$ and $\boldsymbol{x}_{\alpha} \in S^{n_{\alpha}-1}$. Note that the composition map

$$
\circ: S^{k-1} \times S^{n_{1}-1} \times \cdots \times S^{n_{k}-1} \longrightarrow S^{n_{1}+\cdots+n_{k}-1}
$$

describes the $k$-fold join $S^{n_{1}-1} \star \cdots \star S^{n_{k}-1} \cong S^{n_{1}+\cdots+n_{k}-1}$ of the spheres $S^{n_{1}-1}$, $\ldots, S^{n_{k}-1}$. This operad structure on spheres induces an operad structure on (local) coordinates on spheres, since coordinates $\boldsymbol{x}_{0}=\boldsymbol{x}_{0}\left(\varphi_{0,1}, \ldots, \varphi_{0, k-1}\right)$ on $S^{k-1} \subset \mathbb{R}^{k}$ and $\boldsymbol{x}_{\alpha}=\boldsymbol{x}_{\alpha}\left(\varphi_{\alpha, 1}, \ldots, \varphi_{\alpha, n_{\alpha}-1}\right)$ on $S^{n_{\alpha}-1} \subset \mathbb{R}^{n_{\alpha}}$ for $\alpha=1, \ldots, k$ determine 
coordinates

$$
\boldsymbol{x}=\boldsymbol{x}\left(\varphi_{0,1}, \ldots, \varphi_{0, k-1}, \varphi_{1,1}, \ldots, \varphi_{1, n_{1}-1}, \ldots, \varphi_{k, 1}, \ldots, \varphi_{k, n_{k}-1}\right)
$$

on $S^{n_{1}+\cdots+n_{k}-1}$, given by setting

$$
\boldsymbol{x}:=\boldsymbol{x}_{0} \circ\left(\boldsymbol{x}_{1}, \ldots, \boldsymbol{x}_{k}\right) .
$$

The interior of a Stasheff polytope corresponds to elliptic coordinates and its faces are products of Stasheff polytopes. Therefore we can construct all orthogonal separation coordinates on spheres (modulo isometries) from elliptic coordinates by composing them in a recursive manner via the operad composition (6.2). Just start with trivial coordinates $\boldsymbol{x}(\varnothing)=1$ on a certain number of zero dimensional spheres $S^{0}$ and take elliptic coordinates for $\boldsymbol{x}_{0}$ in each step. The different choices one has when iterating this composition are given by the trees labelling the corresponding separation coordinates. That is, the rooted trees describe the hierarchy of iterated decompositions of a sphere as joins of lower dimensional spheres. This parallels the decomposition of the faces of a Stasheff polytope into products of lower dimensional Stasheff polytopes.

Note that the construction (2.13) of Vilenkin's polyspherical coordinates on $S^{n-1}$ corresponds to the special case $k=2$ of the above construction, starting from trivial coordinates $\boldsymbol{x}(\varnothing)=1$ on $n$ copies of $S^{0}$ and using the (elliptic) coordinates $\boldsymbol{x}_{0}(\varphi)=(\cos \varphi, \sin \varphi)$ on $S^{k-1}=S^{1}$ in each step.

Moreover, this operad structure on separation coordinates also explains Kalnins \& Miller's graphical procedure [13]. Namely, adding in an "irreducible block" a leaf to each box which is not joined to another block and replacing each irreducible block by a node, the graphs in [13] become the trees arising from the operad structure.

6.3. Constructing Stäckel systems via the mosaic operad. We now explain how this operad structure manifests itself on the level of Stäckel systems. To this end, let $I_{1} \cup \cdots \cup I_{k}=I$ be a partition of $I=\{1, \ldots, n\}$ with $\left|I_{\alpha}\right|=: n_{\alpha}$ and set $I_{0}:=\{1, \ldots, k\}$. We denote by $\mathfrak{d}_{n}^{1}$ the space of Killing tensors on $S^{n-1}$ with diagonal algebraic curvature tensor and define the injections

$$
\begin{array}{llll}
\iota_{0}: & \mathfrak{d}_{k}^{1} \hookrightarrow \mathfrak{d}_{n_{1}+\cdots+n_{k}}^{1} & \iota_{0}\left(K_{\alpha \beta}\right):=\sum_{a \in I_{\alpha}, b \in I_{\beta}} K_{a b} & \alpha, \beta \in I_{0} \\
\iota_{\alpha}: & \mathfrak{d}_{n_{\alpha}}^{1} \hookrightarrow \mathfrak{d}_{n_{1}+\cdots+n_{k}}^{1} & \iota_{\alpha}\left(K_{i j}\right):=K_{i j} & i, j \in I_{\alpha} .
\end{array}
$$

Proposition 5. Let $\Sigma_{0}$ be a Stäckel system on $S^{k-1}$ and $\Sigma_{\alpha}$ be Stäckel systems on $S^{n_{\alpha}-1}$ for $\alpha=1, \ldots, k$, all consisting of Killing tensors with diagonal algebraic curvature tensor. Then

$$
\Sigma_{0} \circ\left(\Sigma_{1}, \ldots, \Sigma_{k}\right):=\iota_{0}\left(\Sigma_{0}\right) \oplus \iota_{1}\left(\Sigma_{1}\right) \oplus \cdots \oplus \iota_{k}\left(\Sigma_{k}\right)
$$

is a Stäckel system on $S^{n_{1}+\cdots+n_{k}-1}$. Moreover, this operation together with the $S_{n}$-action (2.15) defines an operad structure on those Stäckel systems on $S^{n-1}$ that consist of Killing tensors with diagonal algebraic curvature tensor.

Proof. First observe that the sum on the right hand side of (6.3) is indeed a direct sum. Hence its dimension is $n_{1}+\cdots+n_{k}-1$. By Definition 3 and Remark 3, we have to show that all Killing tensors in this subspace commute. That is, a Killing tensor from $\iota_{p}\left(\Sigma_{p}\right)$ and another one from $\iota_{q}\left(\Sigma_{q}\right)$ commute for all $p, q=$ $0,1, \ldots, k$. For $p, q \neq 0$ this is evident. For $p=q=0$ one readily checks that the 
inclusion $\iota_{0}$ preserves the relations (2.7) and hence maps commuting Killing tensors to commuting Killing tensors. In the remaining case $p \neq 0=q$ the commutator

$$
\left[\iota_{p}\left(K_{i j}\right), \iota_{0}\left(K_{\alpha \beta}\right)\right]=\left[K_{i j}, \sum_{a \in I_{\alpha}, b \in I_{\beta}} K_{a b}\right] \quad i, j \in I_{p}, \quad \alpha \neq \beta \in I_{0}
$$

is zero unless $p=\alpha$ or $p=\beta$. But if $p=\alpha$, the sum over $a \in I_{\alpha}$ only contributes non-zero terms for $a=i$ and $a=j$. So the above commutator reduces to

$$
\sum_{b \in I_{\beta}}\left[K_{i j}, K_{i b}+K_{j b}\right]=0
$$

due to the relations $(2.7)$, and similarly for $p=\beta$. This proves that (6.3) is a Stäckel system.

To check that this composition defines an operad is straightforward. The identity element is the empty Stäckel system on $S^{0}$ and equivariance is obvious. Associativity can be shown by taking subdivisions $I_{\alpha}=I_{\alpha 1} \cup \cdots \cup I_{\alpha k_{\alpha}}$ of $I_{\alpha}$ for all $\alpha \in I_{0}$ and considering the corresponding inclusions for Killing tensors. The details will be left to the reader.

To give an example, let us construct the Stäckel system for standard spherical coordinates on $S^{n-1}$ by choosing $k=2$ with the Stäckel system $\Sigma_{0}$ on $S^{k-1}=S^{1}$ spanned by $K_{12}$, starting from empty Stäckel systems on $n$ copies of $S^{0}$ and taking $n_{2}=1$ in each step. This yields the Stäckel system spanned by

$$
K_{12}, \quad K_{13}+K_{23}, \quad K_{14}+K_{24}+K_{34}, \quad \ldots
$$

and shows that the Jucys-Murphy subalgebras (3.4) in the Kohno-Drinfeld Lie algebra correspond to standard spherical coordinates.

\section{OutLOOK}

We have shown that the theory of Deligne-Mumford-Knudsen moduli spaces and Stasheff polytopes provides the right framework for the classification and construction of all orthogonal separation coordinates on spheres. In particular, we elucidated the natural algebro-geometric structure of the parameter space classifying isometry classes of separation coordinates, which for a long time had only been known as a mere set, and gave a precise description of its topology.

It would be very interesting to see if the same approach will work in a more general situation. In particular, one can use the algebraic approach of [26] to study the orthogonal separation coordinates for all (pseudo-)Riemannian constant curvature manifolds, such as hyperbolic space $\mathbb{H}^{n}$. The question is whether the corresponding moduli spaces of separation coordinates are related to any known algebro-geometric moduli spaces or families of polyhedra. This will be subject for future research.

\section{ACKNowledgements}

Both of us would like to thank the Hausdorff Research Institute for Mathematics in Bonn for its hospitality in March 2012, when this work was essentially done. We are also grateful to Prof. S. Devadoss, who attracted our attention to the paper [8], and to Prof. J. Stasheff for his very useful comments. 


\section{REFERENCES}

[1] L. Aguirre, G. Felder, A.P. Veselov Gaudin subalgebras and stable rational curves. Compositio Math. 147 (2011), no. 5, 1463-1478.

[2] S. Benenti Orthogonal separable dynamical systems. Differential geometry and its applications (Opava, 1992), 163-184, Math. Publ., 1, Silesian Univ. Opava, Opava, 1993.

[3] A.V. Bolsinov and V.S. Matveev Geometrical interpretation of Benenti systems. J. Geom. Phys. (2003) 44, no. 4, 489-506.

[4] C.P. Boyer, E.G. Kalnins, P. Winternitz Separation of variables for the Hamilton-Jacobi equation on complex projective spaces. SIAM J. Math. Anal. 16 (1985), no. 1, 93-109.

[5] M. Crampin Conformal Killing tensors with vanishing torsion and the separation of variables in the Hamilton-Jacobi equation. Diff. Geom. and its Appl. 18 (2003), no. 1, 87-102.

[6] M. Davis, T. Januszkiewicz, R. Scott Nonpositive curvature of blowups. Selecta Math. 4 (1998), no. 4, 491-547.

[7] S.L. Devadoss Tesselations of moduli spaces and the mosaic operad. Contemp. Math. 239 (1999), 91-114.

[8] S.L. Devadoss and R.C. Read Cellular structures determined by polygons and trees. Annals of Combinatorics, 5 (2001), 71-98.

[9] S. Devadoss A realization of graph associahedra. Discrete Math. 309 (2009), no. 1, 271-276.

[10] L.P. Eisenhart Separable systems of Stäckel. Annals of Math. 35 (1934), 284-305.

[11] P. Etingof, A. Henriques, J. Kamnitzer, E. Rains The cohomology ring of the real locus of the moduli space of stable curves of genus 0 with marked points. Annals of Math. 171 (2010), $731-777$.

[12] C.G.J. Jacobi Vorlesungen über Dynamik. In C.G.J. Jacobi's Gesammelte Werke (in German), Berlin: G. Reimer (1881).

[13] E.G. Kalnins and W. Miller Jr. Separation of variables on $n$-dimensional Riemannian manifolds. I. The $n$-sphere $S_{n}$ and Euclidean n-space $R_{n}$. J. Math. Phys. 27 (1986), 1721-1736.

[14] E.G. Kalnins, W. Miller and G.J. Reid Separation of variables for Riemannian spaces of constant curvature. I. Orthogonal separable coordinates for $S_{n C}$ and $E_{n C}$. Proc. Roy. Soc. London Ser. A 394 (1984), no. 1806, 183-206.

[15] M.M. Kapranov The permutoassociahedron, Mac Lane's coherence theorem and asymptotic zones for the KZ equation. J. Pure Appl. Algebra 85 (1993), no. 2, 119-142.

[16] F.F. Knudsen The projectivity of the moduli space of stable curves. II. The stacks $M_{g, n}$. Math. Scand. 52 (1983), 161-199.

[17] G. Lamé Sur les surfaces isothermes dans les corps homogènes en équilibre de température. J. Math. Pures Appl. 2 (1837), 147-188.

[18] C. Lee The associahedron and triangulations of the n-gon. European J. Combin. 10 (1989), no. $6,551-560$.

[19] T. Levi-Civita Sulla integrazione della equazione de Hamilton Jacobi per separazione de variabili. Math. Ann. 59 (1904), 383-397.

[20] V.S. Matveev, P. Mounoud Gallot-Tanno theorem for closed incomplete pseudo-Riemannian manifolds and applications. Ann. Global Anal. and Geom. 38 (2010), no. 3, 259-271.

[21] R.G. McLenaghan, R. Milson, R.G. Smirnov Killing tensors as irreducible representations of the general linear group. C. R. Acad. Sci. Paris 339 (2004), 621-624.

[22] F. Müller-Hoissen, J.M. Pallo, J. Stasheff (Editors) Associahedra, Tamari Lattices and Related Structures. Tamari Memorial Festschrift. Progress in Mathematics 299, Birkhäuser, 2012.

[23] C. Neumann De problemate quodam mechanico, quod ad primam integralium ultraellipticorum classem revocatur. J. Reine Angew. Math. 56 (1859), 46-63.

[24] M.N. Olevskiı Triorthogonal systems in spaces of constant curvature in which the equation $\Delta_{2} u+\lambda u=0$ admits separation of variables. Math. Sbornik 27 (1950), 379-426.

[25] K. Schöbel Algebraic integrability conditions for Killing tensors on constant sectional curvature manifolds. Journal of Geometry and Physics 62 (2012), no. 5, 1013-1037.

[26] K. Schöbel The variety of integrable Killing tensors on the 3-sphere. arXiv preprint, arxiv:1205.6227.

[27] P. Stäckel Die Integration der Hamilton-Jacobischen Differentialgleichung mittelst Separation der Variabeln. (1891), Habilitationsschrift Universität Halle. 
[28] J. Stasheff Homotopy associativity of H-spaces. I, II. Trans. Amer. Math. Soc. 108 (1963), $275-312$

[29] A.M. Vershik, A.Yu. Okounkov A new approach to representation theory of symmetric groups. II. Zap. Nauchn. Sem. S.-Peterburg. Otdel. Mat. Inst. Steklov (POMI), 307 (2004), 57-98. English transl.: J. Math. Sci. 131 (2005), 5471-5494.

[30] N.Ya. Vilenkin Special functions and group representation theory. Nauka Publ. Comp., Moscow, 1965 (in Russian).

[31] N.Ja. Vilenkin and A.U. Klimyk Representation of Lie Groups and Special Functions. Volume 2: Class I Representations, Special Functions, and Integral Transforms. Kluwer Academic Publishers (1993).

[32] OEIS Foundation Inc. (2011), Sequence A001190, The On-Line Encyclopedia of Integer Sequences, http://oeis.org/A001190.

[33] OEIS Foundation Inc. (2011), Sequence A032132, The On-Line Encyclopedia of Integer Sequences, http://oeis.org/A032132.

Friedrich-Schiller-Universität Jena, FAkUltät FÜr Mathematik Und Informatik, ERnstAbbe-Platz 2, 07743 Jena, Germany

E-mail address: konrad.schoebel@uni-jena.de

Department of Mathematical Sciences, Loughborough University, Loughborough, Leicestershire, LE11 3TU, UK and Moscow State University, Russia

E-mail address: A.P.Veselov@lboro.ac.uk 